
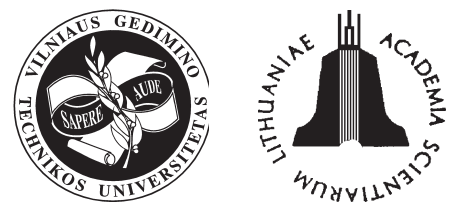

ISSN 1648-4142 TRANSPORT

http:/www.vtu.lt/english/editions

TRANSPORT - 2005, Vol XX, No 2,83-89

\title{
AN INPUT-OUTPUT MATHEMATICAL MODEL FOR MEASURING THE PRODUCTIVITY OF ROAD-TRANSPORT COMPANIES
}

\author{
Sunday Ayoola Oke \\ Dept of Mechanical Engineering, University of Lagos, Akoka-Yaba, Lagos, Nigeria \\ Tel: (234-01) 4938637-9 Ext. 1114, E-mail: sa_oke@yahoo.com
}

Received 2004-08-01; accepted 2005-01-25

\begin{abstract}
This paper presents a mathematical model for measuring the productivity of a transport company that is engaged in commercial transport activities. The model is input-output based and takes into account the inflation factor that the system resources are subjected due to the changes in prices of these input items. Drawing from the principles of composite variable formulation in differential calculus, the productivity framework was formulated to mimic Maclaurin series function in mathematical sciences. Based on this boundary conditions were set in order to have a definite model that measures the productivity of the transport system. A hypothetical case study was formulated and solved to demonstrate the practical applicability of the model proposed. Arising from this there is a number of graphical presentations of the results that validate the feasibility of measuring the productivity of the transport company using the model developed.
\end{abstract}

Keywords: productivity measurement, transport company, mathematical model, vehicle service, input, output.

\section{Introduction}

In achieving continuous system improvement in transport systems a number of crucial issues need to be considered: the hiring and maintenance of skilled professionals is of prime importance. Therefore, adequate plans for employee succession activities must be put in place. Funds must also be available. Financial resources must be available for activities to be carried out at budgeted time and resources. However, there is need to measure the utilization of these scarce resources. Productivity is one effective tool utilized in this regard.

Defined as the ratio of output of the transport system to the input resources utilized to achieve these outputs productivity has proved to be an effective tool towards achieving global competitiveness in transport system operations $[8,11]$. Despite this, no documentation seems to have been made on the conceptualization of the productivity problem in a way that would incorporate the inflation factor in any transport system. The need to close a wide gap has motivated the present study.

We developed a mathematical model in such a way that productivity is tracked in a series of behavioural pattern. The application of Maclaurin series is used in this regard. Transport system productivity is defined in such a way that it encompasses the inflation index, the unit sales price of transport system services, the output quantity of the transport system and the unit cost price of the input resources utilized to attain the output.

A large number of research activities has been carried out into service and manufacturing productivity $[9,10]$. Studies on service system productivity include those of Stainer [11], Gupta [5], Sigala, and Awuku [1]. Babbar et al. [2] assesses institutional and individual research productivity empirically by reviewing publications in 21 leading operations management (OM) journals over the 12-year period.

Office productivity was investigated by Leaman [7] with a question on whether people's productivity in office is affected by environmental conditions, such as heat, lighting, ventilation and noise. Findings point to individual's perception that uncomfortable working conditions affect their productivity.

Halachmi [6] carried out a study on government productivity to examine the changing nature of governments through performance measurement and management as governments attempt to balance accountability for the resources they manage with flexibility in terms of the services they provide.

Numerous articles on manufacturing-related productivity exist. Goh [4] studied how statistical literacy for manufacturing productivity could be raised by attaining proficiency in the statistical approach in an organization. 
Fisher [3] measures the productivity using standard cost accounting the information based on the standard total productivity model. Critical factors in the approach are: the use of business goals and the determination of effective output measures.

In conclusion, since no documentation exists on productivity in the transport sector that has incorporated an inflation factor, the current paper is justified.

\section{Transport company productivity measurement model}

The worldwide definition of productivity involves expressing the productivity value in terms of the quantified output obtained from any system and the input resource utilized for the smooth running of the operations of the system.

Mathematically,

Transport company productivity $(\mathrm{P})$

$=$ Output of the transport system

$=$ Input resources utilised to produce the output

Thus, if $\mathrm{P}$ represents the productivity of the transport company, then the expression that represents productivity is:

$$
P=\frac{Q U}{I C\left(1-n_{O}\right)^{t}} .
$$

The components of this expression are defined as follows:

$Q$ represents the quantity of output of the transport system in terms of the number of passengers or goods transported for the time period that the productivity is to be measured. Thus, the transport system may refer to a system of different mediums of moving people and goods efficiently such as air, land, and sea. This may include the use of commercial airplanes, vehicles, railways, and ships. However, for the purpose of simplicity of modelling we shall be limited to modelling fleet of vehicles that transport goods and people from one location to another. Thus, the output in this respect may be the number of people conveyed from a source to a destination taking note of the unit price paid by each passenger and the number of trips observed by the vehicle. Such vehicles may be dedicated to either passenger/goods conveyance or a combination of both.

Specifically, $U$ represents the sales price of services offered by the transport company to the public. In addition, $I$ refers to the quantity of inputs utilised to attain the output, while $C$ refers to the unit cost price of input utilised in the production of services. In addition, $\mathrm{n}_{0}$ represents the inflation index.
The starting point for the model for the model formulation is to split the equation (1) into three different components, represented as:

$$
Y=\frac{Q}{C}, \quad X=\frac{U}{I}, \quad \text { and } T=\frac{1}{\left(1-n_{0}\right)^{\gamma}} .
$$

Thus, we have a new equation as:

$$
P=\frac{Q}{C} \times \frac{U}{I} \times \frac{1}{\left(1-n_{0}\right)^{t}} .
$$

The functional representative of the problem is:

$P=Y(Q) X(I) T(t)$.

This is based on the assumption that we let

$$
Y=\frac{Q}{C}, \quad X=\frac{U}{I}, \quad \text { and } T=\frac{1}{\left(1-n_{0}\right)^{t}} \text {. }
$$

Applying the second order partial differential equation principles we have a new expression as:

$$
\frac{\partial^{2} p}{\partial Q^{2}}+\frac{\partial^{2} p}{\partial I^{2}}=\frac{1 . \partial^{2} T}{\mathrm{e}^{2} T \partial t^{2}}
$$

(e is a constant).

This partial differential equation could be converted to a second order differential equation as:

$$
\frac{1}{Y} \frac{d^{2} Y}{d Q^{2}}+\frac{1}{x} \frac{d^{2} x}{d T^{2}}=\frac{1}{\mathrm{e}^{2} T} \frac{d^{2} T}{d t^{2}} .
$$

An equation is developed and compared with equation (5) so as to apply Maclaurin series. The equation is developed:

$$
\frac{1}{Y} \frac{d^{2} Y}{d Q^{2}}+m^{2}=0(\mathrm{~m} \text { is a constant })
$$

Equation (6) could be transformed as:

$$
\frac{d^{2} Y}{d Q^{2}}+m^{2} Y=0 .
$$

The general mathematical solution to the equation formulated in (7) is as stated below:

$$
Y=A \sin (m Q)+B \cos (m Q)=\frac{\mathrm{Q}}{\mathrm{C}} .
$$

The right hand side of equation (8) suggests that the first component of the productivity equation in (2) is considered. To get the particular solution we consider the boundary conditions at $\mathrm{Q}=0$ and $\mathrm{Y}=$ 0 . This implies that $\mathrm{Y}=0=\mathrm{B} \operatorname{Cos}(\mathrm{mQ})=0$. From here we obtain $B$ to be 0 . Now, if $B$ is initially said to be 0 in the equation (8), then: 
$Y=A \sin (m Q)=\frac{Q}{C}$.

Now changing the boundary conditions to $\mathrm{Q}=$ 1 , we have the expression

$$
Y=A \sin (m)=\frac{1}{C} .
$$

Therefore, $\left(\frac{d Y}{d Q}\right)=A m \cos (m)=\frac{1}{C}$.

Now dividing equation (10) by equation (11), we have:

$\frac{A \sin (m)}{A m \cos (m)}=\frac{1 / C}{1 / C}$.

Which gives

$$
\frac{\sin (m)}{m \cos (m)}=1
$$

From our knowledge of Maclaurins series using Maclaurin series

$$
\sin (m)=\left(m-\frac{m^{3}}{6}\right)
$$

and

$$
\cos (m)=\left(1-\frac{m^{3}}{2}+\frac{m^{4}}{24}\right)
$$

Now relating equations (14) and (15) to equation (13), we have:

$$
\frac{\sin (m)}{\cos (m)}=\frac{m-\frac{m^{3}}{6}}{\left(1-\frac{m^{2}}{2}+\frac{m^{4}}{24}\right)}=1 .
$$

From equation (16) we obtain $m=\sqrt{8}$.

Now substituting for $\mathrm{m}$ in equation (9), i.e. $Y=A \sin (m Q)$, we have:

$$
Y=A \sin \sqrt{8} Q \text {. }
$$

Now differenciating $\mathrm{Y}$ with respect to $\mathrm{Q}$,

$$
\begin{aligned}
& \frac{d Y}{d Q}=A \sqrt{8} \operatorname{Cos} \sqrt{8} Q=\frac{1}{C} . \\
& A t=0, \quad A \sqrt{8}=\frac{1}{C}, \quad A=\frac{1}{C \sqrt{8}} .
\end{aligned}
$$

Now substituting for $A$ in equation (9), we have:

$$
Y=\frac{1}{C \sqrt{8}} \operatorname{Sin} \sqrt{8} Q
$$

Going back to equation (5), it would be observed that the first component of the equation i.e. $\frac{1}{Y} \frac{d^{2} Y}{d Q^{2}}$ is modelled to follow Maclaurins series. If

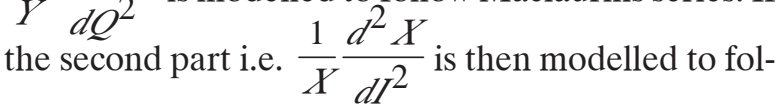
low Maclaurins series, then we have a new equation:

$\frac{1}{X} \frac{d^{2} X}{d I^{2}}+n^{2}=0$ ( $n$ is a constant $)$.

By multiplying with a factor " $X$ ", we have:

$$
\frac{d^{2} X}{d I^{2}}+n^{2} X=0
$$

The function for equation (22) can be written as:

$$
X=D \sin (n I)+E \cos (n I)=\frac{U}{I} .
$$

If we let $D=E=l$, then

$$
\begin{aligned}
& X=\lambda(\sin (n I)+\cos (n I))=\frac{U}{I} \\
& \text { At } I=1, X=\mathrm{U} \\
& X=\lambda(\sin (n)+\cos (n))=U \\
& \text { But }\left(\frac{d X}{d I}\right)_{I=1}=\lambda(n \sin (n)-\cos (n))=-U .
\end{aligned}
$$

Dividing equation (25) by (26), we have:

$$
\frac{\sin (n)+\cos (n)}{n(\cos (n)-\sin (n))}=-1
$$

Thus:

$\sin (n)+\cos (n)=n \sin (n)-n \cos (n)$.

By using Maclaurins series we know that

$\sin n \approx n-\frac{n^{3}}{6}$ and $\cos n \approx 1-\frac{n^{2}}{2}$.

By substituting the values of $\sin (n)$ and $\cos (n)$ of equation (29) in equation (28), we have:

$$
\frac{n^{4}}{6}-\frac{-4 n^{3}}{6}-\frac{3 n^{2}}{2}-n^{2}-2 n+1=0 .
$$

If we consider small values of $\mathrm{n}$, therefore

$$
\frac{n^{4}}{6}-\frac{4 n^{3}}{6} \text { will be negligible. }
$$

We would then go ahead to solve the equation 
$-\frac{3 n^{2}}{2}-2 n+1=0$ to obtain the value of $n=0,387$.

Note that if we substitute this value of $\mathrm{n}$ in equation (25), we have a new value for $l$. Thus, we have:

$$
\lambda=\frac{U}{\sin 0,387+\cos 0,387}=0,993 U .
$$

Thus:

$$
X=0,993 U(\sin 0,3871+\cos 0,3871) \text {. }
$$

If we consider the third part of equation (5) which is on the right hand side of the equation and model it as in the previous cases, then we start by supposing that:

$$
\frac{1}{e^{2} T} \frac{D^{2} t}{d t^{2}}+r^{2}=0(r \text { is a constant })
$$

Therefore, the solution becomes

$$
T=D \sin (e r t)+E \cos (e r t)=\left(1-n_{0}\right)^{-t} .
$$

At $t=0, T=1, E=1$.

Thus, we have:

$$
T=D \sin (e r t)+\cos (e r t)=\left(1-n_{0}\right)^{-t} \text {. }
$$

This means that when $T$ is differentiated with respect to $t$, we have:

$$
\begin{gathered}
\left(\frac{d T}{d t}\right)_{t=0}=\text { Der cos(ert) }- \text { er sin }(\text { ert })= \\
\left(-1-n_{0}\right)^{-t} \ln \left(1-n_{0}\right) .
\end{gathered}
$$

Equation (34) reduces to the form in equation (35) below

$$
\begin{aligned}
& T=\operatorname{Der}=-\left(1-n_{0}\right)-t \ln \left(1-n_{0}\right), \\
& \text { but } r=\sqrt{m^{2}+n^{2}}=\sqrt{8+0,15}=2,86 .
\end{aligned}
$$

If we have to substitute the value of $r$ and $e$ in equation (35), we may need to assume $e=1$. Together with the value of $r$ we have a new expression labelled equation (36)

$$
D=-\left(1-n_{0}\right)^{-t} \frac{\ln \left(1-n_{0}\right)}{2,86} .
$$

Now, substituting the expression for $D$ from equation (36) to equation (34), the expression becomes:

$$
T=-\frac{\left(1-n_{0}\right)^{-t}}{2,86} \ln \left(1-n_{0}\right) \sin 182,86 t+\cos 2,86 t \text {. }
$$

Thus,

$$
\begin{aligned}
P= & \frac{1}{c \sqrt{8}} \sin \sqrt{8} \mathrm{Q}(0,993 \mathrm{U}(\sin 0,387 \mathrm{I}+\cos 0,387 \mathrm{I})) \\
& \left(\frac{\left(1-\mathrm{n}_{0}\right)^{-\mathrm{t}}}{2.86} \ln \left(1-\mathrm{n}_{0}\right) \sin 182,86 \mathrm{t}+\cos 2,86 \mathrm{t}\right)
\end{aligned}
$$

Finally, we have:

$$
\begin{aligned}
P= & \frac{0,351 U}{c} \sin \sqrt{8} Q(\sin 0,387 I+\cos 0,387 l) \\
& \left(\frac{\left(1-n_{0}\right)^{-t}}{2.86} n\left(1-n_{0}\right) \sin 182,86 t+\cos 2,86 t\right)
\end{aligned}
$$

What follows is an illustrative case study that demonstrates the practicality of the model.

\section{Case study}

For the purpose of illustration and clarity of the model application we illustrated the application of the model with the use of a case study drawn from TCTC Transport Company in Lagos Nigeria. The company has been in existence for ten years, but has just restructured due to changes in the number and the sophistication of the transport facilities it manages. This government company had just received donations of 1,500 traveling vehicles that convey both human and non-human elements across the country. Six months into the assumption of the office which coincide with the time that the donations were made, the government of the state has requested for a detailed statement of the productivity of the transport organization for two months from the manager of TCTC.

As a way of resolving the problem, the data on the activities of the company were used concerning the prices attached to transport services and the number of passengers each vehicle carries at a particular instance and the weight of goods carried for the different routes. These data need to be complimented with that of the quality and unit prices of the various input resources utilized for the transportation activities. Based on this information a computer program was developed that computes the productivity of the organization as defined in equation (39). The program developed is as stated below: to obtain the calculated value of productivity random numbers are first generated and used to test the work ability of the model under different circumstances.

Results generated from the development of the computer programme are presented in Table.

In order to demonstrate the behaviour of the various variables that are captured in the model, the results obtained were plotted graphically over the 
periods of 12 months. Variations in $t, Z, I, n, Q, U$, and $P$ were plotted as line graphs as shown in Fig 1 . Another point of interest is to understand the changes in productivity and an inflation factor over 12-month period. The graph for this is shown in Fig 2. A con- cern is to study the effects of changes of the input quantities and price of input over 12-month period. This is displayed in Fig 3. In Fig 4 we have the changes in value of the output over the period of the test.

Results generated from the development of the computer programme

\begin{tabular}{cccrrrrrr}
\hline Month & $\mathrm{t}$ & $\mathrm{z}$ & $\mathrm{I}$ & $\mathrm{C}$ & $\mathrm{n}$ & $\mathrm{Q}$ & $\mathrm{U}$ & $\mathrm{P}$ \\
\hline 1 & .5873519 & .7066861 & 5 & 313.2331 & .446268 & 21 & 69.78876 & $-1.558046 \mathrm{E}-03$ \\
2 & 1.186813 & .1091816 & 51 & 122.2464 & .8452781 & 47.87303 & 87 & $-5.316107 \mathrm{E}-02$ \\
3 & .4510369 & .1843805 & 60 & 513.4389 & .9764491 & 70 & 81.86306 & $-3.758626 \mathrm{E}-04$ \\
4 & .1369649 & .8188099 & 2 & 650.3797 & .767653 & 2 & 36.03609 & $-5.797413 \mathrm{E}-03$ \\
5 & .5337111 & .7412128 & 0 & 958.3954 & .4294213 & 8 & 16.21495 & $-1.592213 \mathrm{E}-04$ \\
6 & .6101571 & .9145923 & 50 & 852.4893 & .1361169 & 85 & 15.8546 & $-1.225355 \mathrm{E}-04$ \\
7 & 1.662356 & .6877908 & 19 & 943.4751 & .2015994 & 60 & 50.21745 & $-3.397961 \mathrm{E}-05$ \\
8 & 2.956251 & .3348614 & 9 & 580.6848 & .3093184 & 13 & 92.34255 & $-1.184773 \mathrm{E}-02$ \\
9 & .6192182 & .4209653 & 42 & 170.2483 & .7527249 & 43 & 42.68474 & $9.163597 \mathrm{E}-03$ \\
10 & 1.507155 & .8089356 & 8 & 593.353 & .1312381 & 16 & 8.81604 & $5.065117 \mathrm{E}-04$ \\
11 & 1.292246 & .3624585 & 13 & 76.11603 & .5440329 & 30 & 79.29971 & $-1.628008 \mathrm{E}-03$ \\
12 & .368314 & .5945884 & 9 & 691.0934 & .7562298 & 24 & 74.59893 & $2.103868 \mathrm{E}-02$ \\
\hline
\end{tabular}

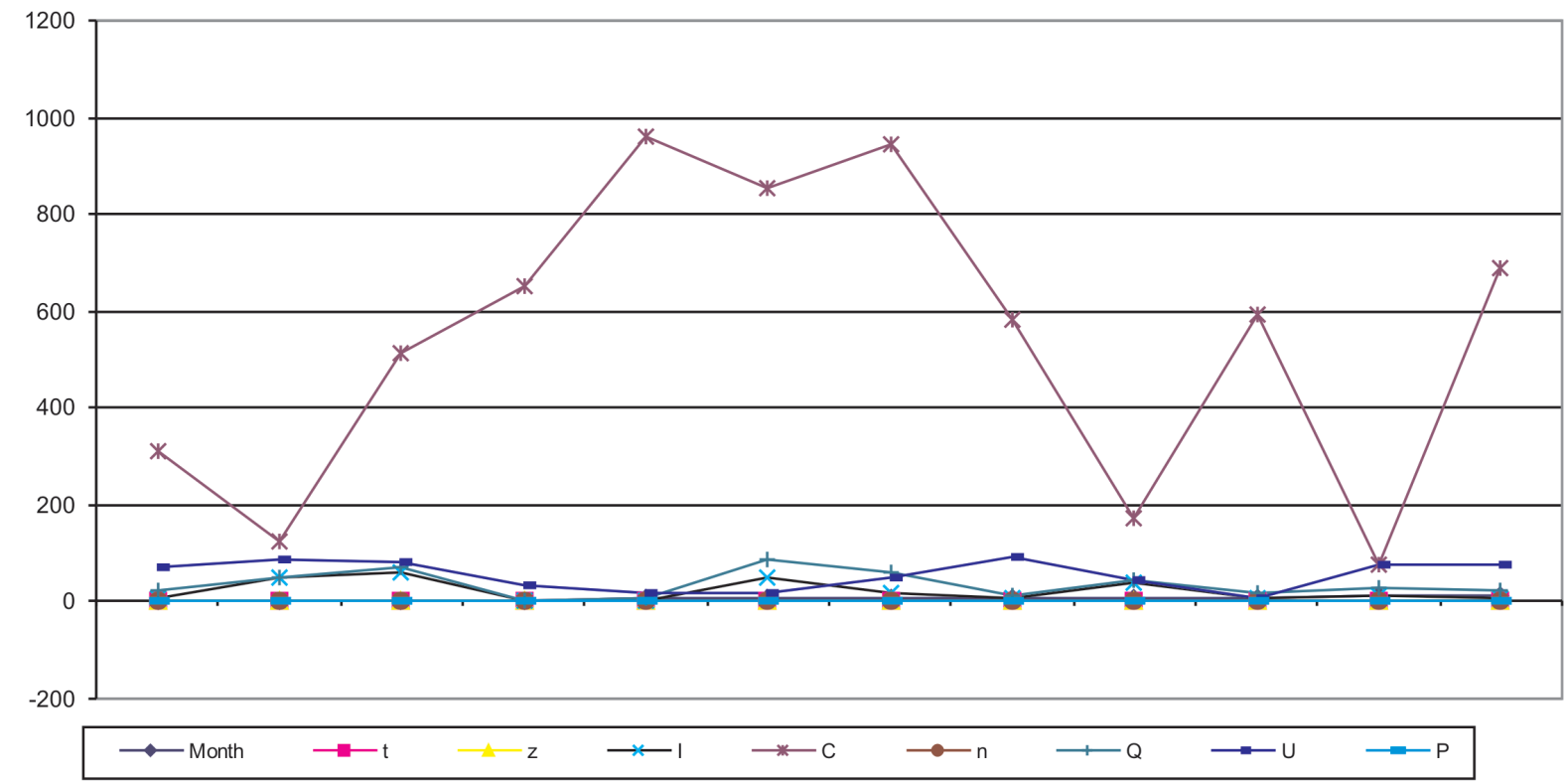

Fig 1. Productivity indices for TCTC Transport Company (12 months) 


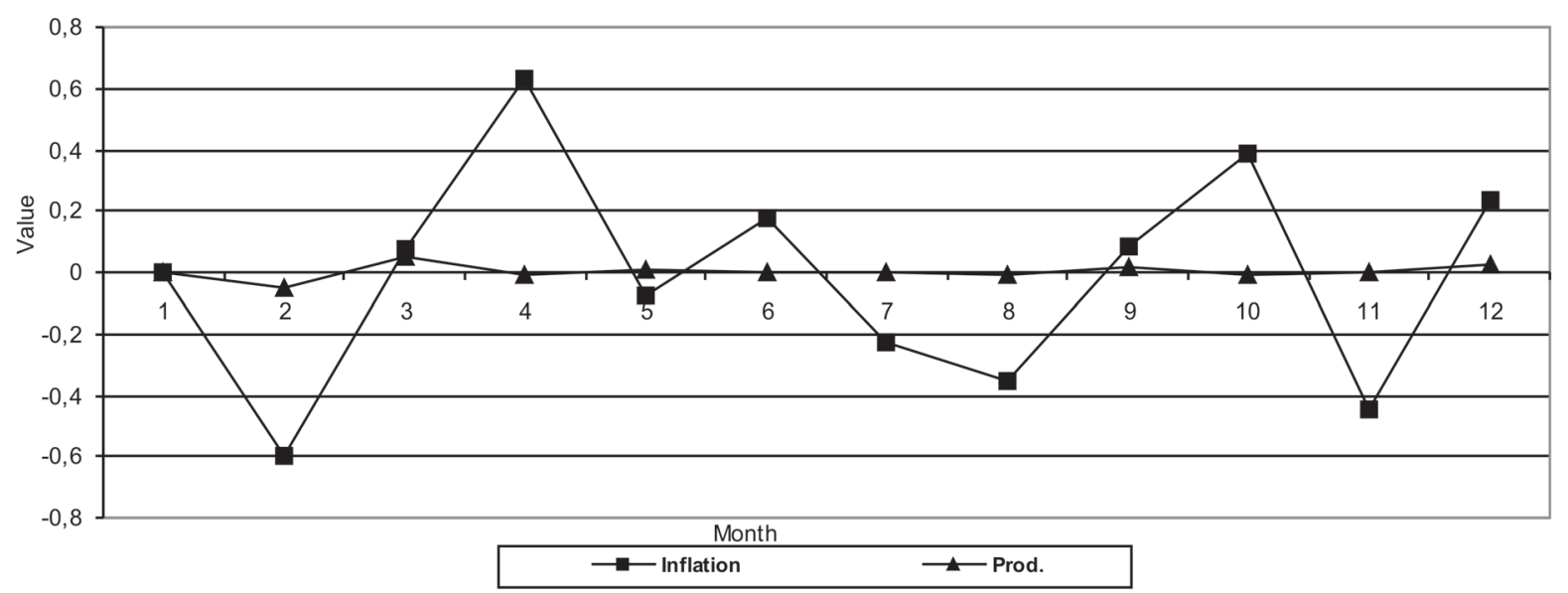

Fig 2. Periodic changes of an inflation factor and productivity

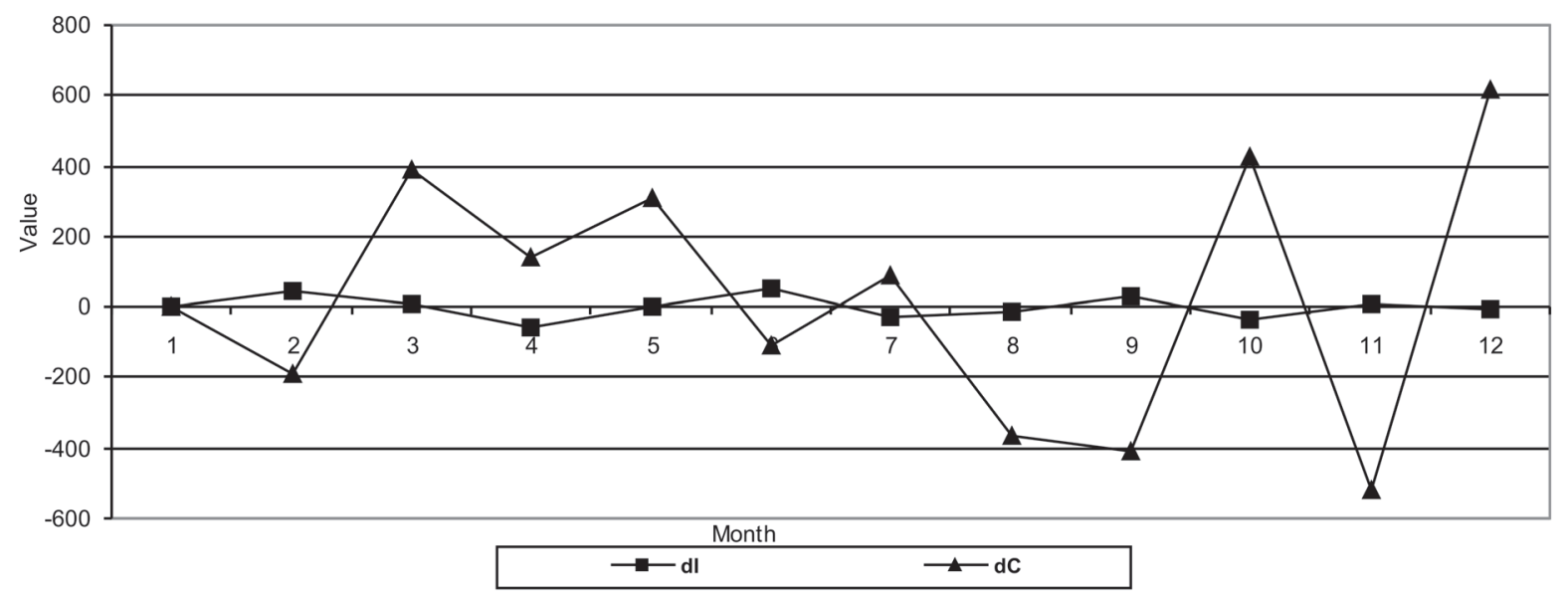

Fig 3. Changes of value of the input resources over the measurement period

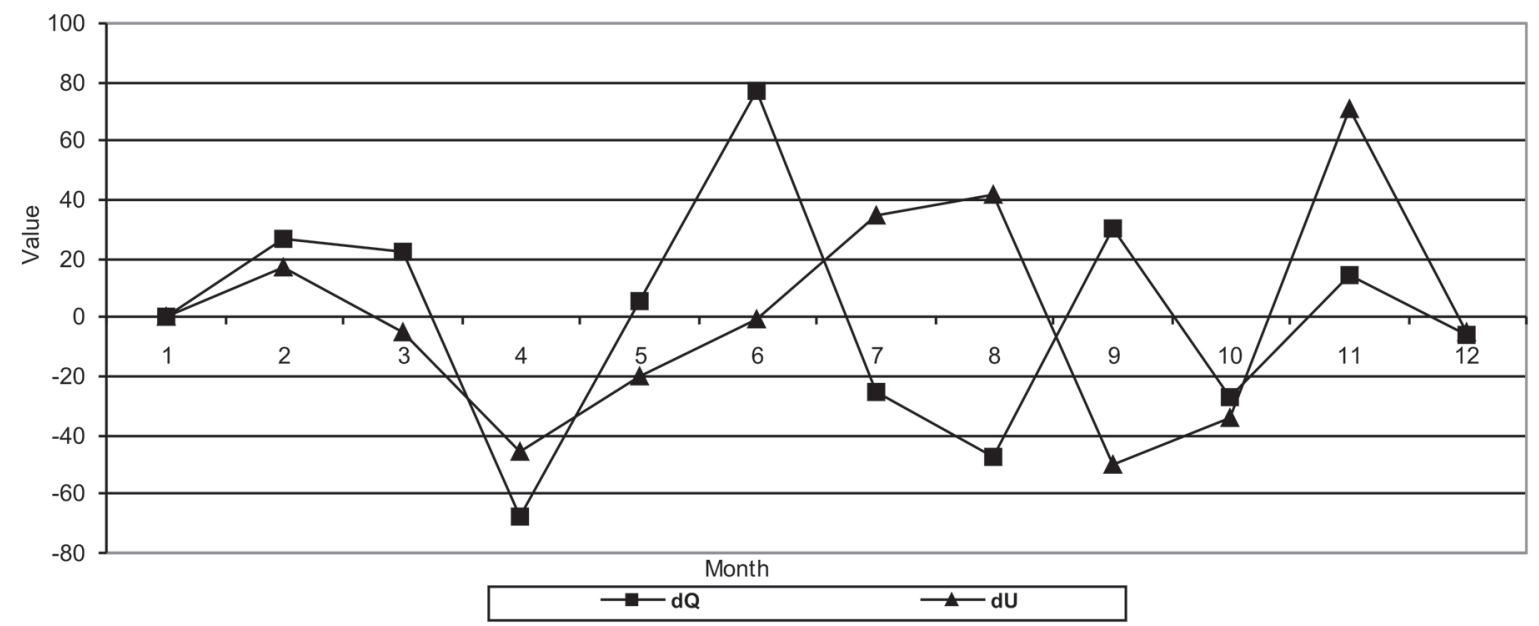

Fig 4. Changes of value of the output 


\section{Conclusion}

In this work productivity is used to diagnosis transport company's performance ailments. An attempt was made to quantify the output of the transport system with a corresponding quantified input for the measurement of performance. In particular, Maclaurin's series principle in mathematics is adopted due to the pattern observed in the model. With this result we hopefully add new methodology of measuring productivity to the decision-maker's tool kit. The model serves some other usefulness.

During labour disputes, this valuable tool may act as scientific support for conflict resolution since it will provide empirical data to support the decision. The model proposed could be used for bench-marking analysis in transport companies.

Interestingly this study has opened up some new areas for investigation. Since no claim has been made concerning the model testing, an immediate test should be carried out to understand the degree of responsiveness of model parameters to changes in value under different conditions. It can then be concluded from the results obtained that some of the model parameters are responsive to changes while others are not. Another area of investigation is the test of robustness of the model. Future research can also consider the incorporation of other material not considered in this work. Two important considerations are: (i) the transport company's capacity changes and (ii) the technological changes within the transport company. The first consideration above (i.e. capacity changes) may be due to organizational restructuring which may be in terms of expansion or shrinkage. The other consideration of technological changes also follows the same line of argument as the first consideration. It should be noted that the analysis with the use of manual is tasky, repetitive and sometimes uninteresting. We therefore suggest the incorporation of a computer program that will automate the productivity measurement system in the transport company. With this signals could be given for poor performance so that correct actions are taken.

\section{References}

1. Awuku, O. S. Productivity in university libraries of developing countries: A West and Southern African perspective. Library Management, Vol 16, No 3, 1995, p. 24-33.

2. Babbar, S.; Prasad, S.; Tata, J. An empirical assessment of institutional and individual research productivity in international operations management. International Journal of Operations \& Production Management, Vol 20, No 12, 2000, p. 1392-1410.

3. Fisher, T. J. Business productivity measurement using standard cost accounting information, International Journal of Operations \& Production Management, Vol 10, No 8, 1990.

4. Goh, T. N. Raising statistical literacy for manufacturing productivity. International Journal of Quality \& Reliability Management, Vol 7, No 3, 1990.

5. Gupta, A. Productivity measurement in service operations: A case study from the health-care environment. Managing Service Quality, Vol 5, No 5, 1995, p. 31-35.

6. Halachmi, A. Performance measurement and government productivity. Work Study, Vol 51, No 2, 2002, p. 63-73.

7. Leaman, A. Dissatisfaction and office productivity. $\mathrm{Fa}$ cilities, Vol 13, No 2, 1995, p. 13-19.

8. Ray, P. K.; Sahu, S. The measurement and evaluation of white-collar productivity. International Journal of Operations \& Production Management, Vol 9, No 4, 1989.

9. Saha, A. Computer-based total productivity measurement: An indian chemical plant. Industrial Management \& Data Systems, Vol 94, No 7, 1994, p. 3-13.

10. Sedgewick, D. Productivity control - equipment and facilities. Work Study, Vol 41, No 7, 1992.

11. Stainer, A. Logistics - A productivity and performance perspective. Supply Chain Management: An International Journal, Vol 2, No 2, 1997, p. 53-62. 